\title{
Les sondages archéologiques à l'église paroissiale de Gigny-sur-Suran (Jura)
}

David Billoin

\section{(2) OpenEdition}

1 Journals

Édition électronique

URL : https://journals.openedition.org/cem/11891

DOI : 10.4000/cem.11891

ISSN : 1954-3093

\section{Éditeur}

Centre d'études médiévales Saint-Germain d'Auxerre

\section{Édition imprimée}

Pagination : 43-47

ISSN : 1623-5770

\section{Référence électronique}

David Billoin, «Les sondages archéologiques à l'église paroissiale de Gigny-sur-Suran (Jura)», Bulletin du centre d'études médiévales d'Auxerre | BUCEMA [En ligne], 15 | 2011, mis en ligne le 19 août 2011, consulté le 22 septembre 2022. URL : http://journals.openedition.org/cem/11891 ; DOI : https:// doi.org/10.4000/cem.11891

Ce document a été généré automatiquement le 22 septembre 2022.

\section{(c) (1) (2) (2)}

Creative Commons - Attribution - Pas d'Utilisation Commerciale - Partage dans les Mêmes Conditions 4.0 International - CC BY-NC-SA 4.0

https://creativecommons.org/licenses/by-nc-sa/4.0/ 


\section{Les sondages archéologiques à l'église paroissiale de Gigny-sur- Suran (Jura)}

\section{David Billoin}

Le village de Gigny-sur-Suran garde encore l'empreinte de la topographie monastique avec plusieurs bâtiments conservés, dont l'église abbatiale qui en est l'épicentre (fig. 1).

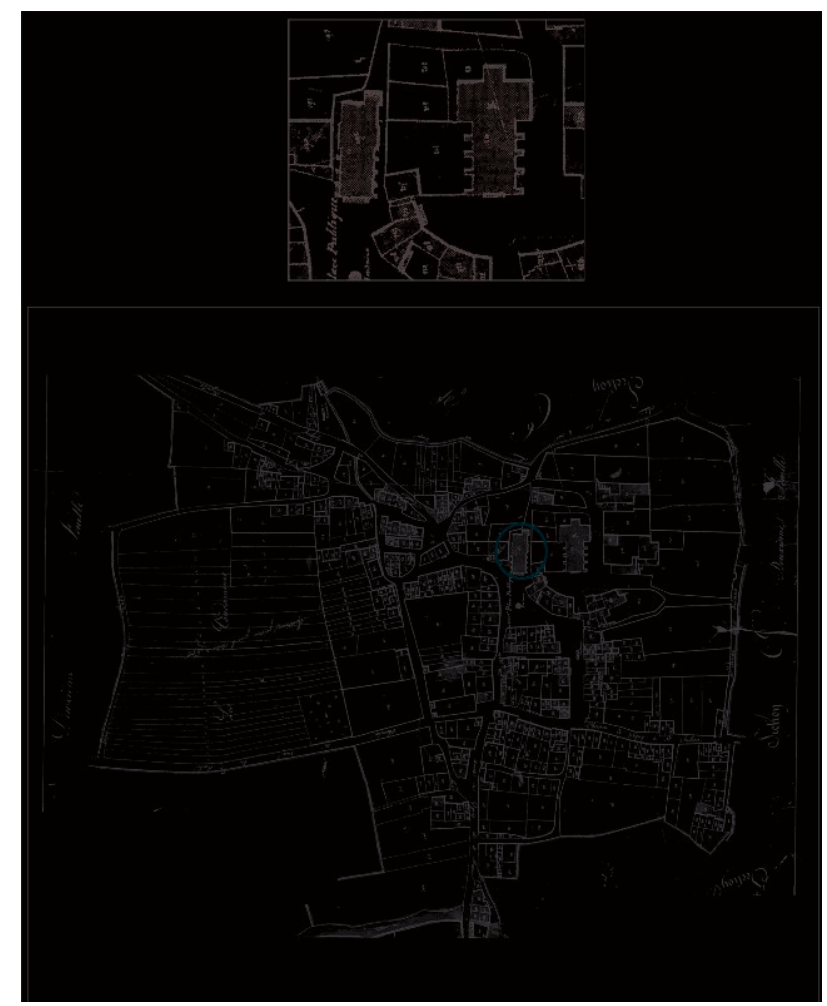

Fig. 1 - Gigny-sur-Suran, localisation de l'église paroissiale sur le cadastre napoléonien (Dao D. Billoin, Inrap). 
Des recherches sont toujours en cours sur cet édifice cultuel, notamment sur le plan primitif, sous la responsabilité de Christian Sapin ${ }^{1}$. L'église paroissiale, quant à elle, est restée en marge des recherches jusqu'en $2006^{2}$. On sait qu'elle est construite vers la fin du XII ${ }^{\mathrm{e}}$ ou au début du XIII ${ }^{\mathrm{e}}$ siècle, car le pape Urbain III a statué que les paroissiens ne pouvaient plus assister aux offices monastiques dans l'église abbatiale. Réparée en 1672, elle tombe ensuite en ruine, pour être interdite en 1770 et démolie en 1774 . A. Rousset en donne une description: "L'église paroissiale primitive était moins grande que celle qui l'a remplacée. Elle était couverte en lave et n'avait, en place du clocher, qu'un campanile avec une petite cloche ${ }^{3}$. " La nouvelle église reconstruite à son emplacement en 1780, sous le double vocable de Notre-Dame de l'Assomption et de Saint-Taurin, devient inutile après la Révolution. L'édifice sert alors de halles pour les foires et la voûte est démolie vers 1840 . Hormis le cadastre napoléonien, seul un croquis au crayon du début du XIX ${ }^{\mathrm{e}}$ siècle montre un aperçu de l'édifice (fig. 2).

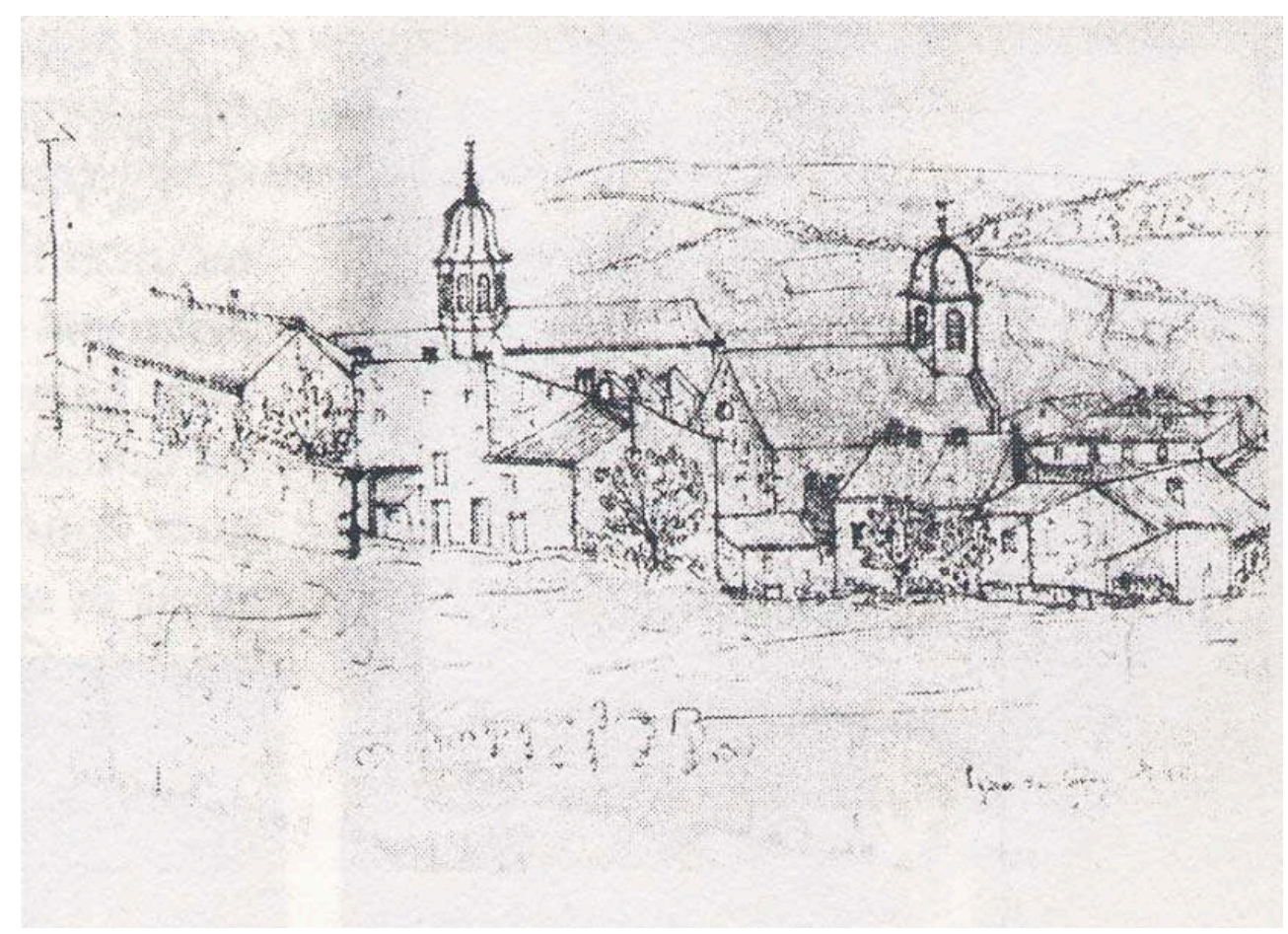

Fig. 2 - Gigny-sur-Suran, croquis au crayon de l'église paroissiale au premier plan, xıx siècle (cl. D. Billoin, Inrap).

3 A. Rousset en donne également une brève description : «Elle se composait d'un clocher couronné par un dôme, d'une nef, d'un transept légèrement prononcé, d'un sanctuaire rectangulaire et d'une sacristie ${ }^{4}$. » Une fouille ponctuelle, menée parallèlement à une surveillance de travaux en 1986, a permis de mettre au jour des portions de mur de la nef de l'édifice, montrant la parfaite superposition de la mairie avec l'église et le cadastre de 1820 .

4 L'agrandissement du bureau de poste, accolé à la mairie, en 2006, a permis de compléter ces informations et de reconnaître une partie de l'église paroissiale primitive ${ }^{5}$. Une fenêtre de sondage de $50 \mathrm{~m}^{2}$ a été ouverte sur ce projet et a atteint le sous-sol géologique, des argiles orangées compactes, à $0,90 \mathrm{~m}$ de profondeur (382,07 m NGF), sous un remblai constitué, pour l'essentiel, de la démolition de l'église 
de la fin du xviII siècle. Deux massifs de maçonnerie, de forme quadrangulaire d'environ $2 \mathrm{~m}$ de côté, ont été identifiés à $6 \mathrm{~m}$ l'un de l'autre (fig. 3 et 4).

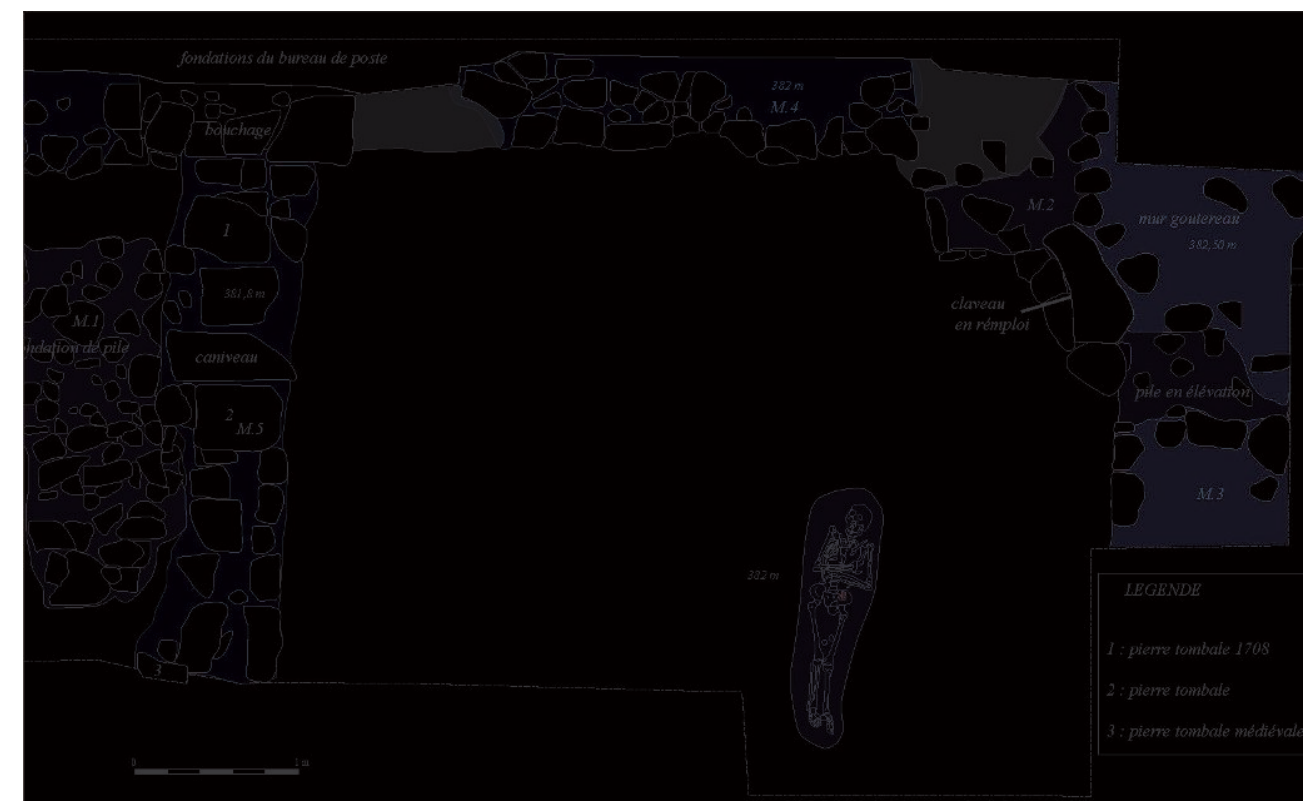

Fig. 3 - Gigny-sur-Suran, relevé des vestiges archéologique du sondage (D. Billoin et P. Haut, Inrap).

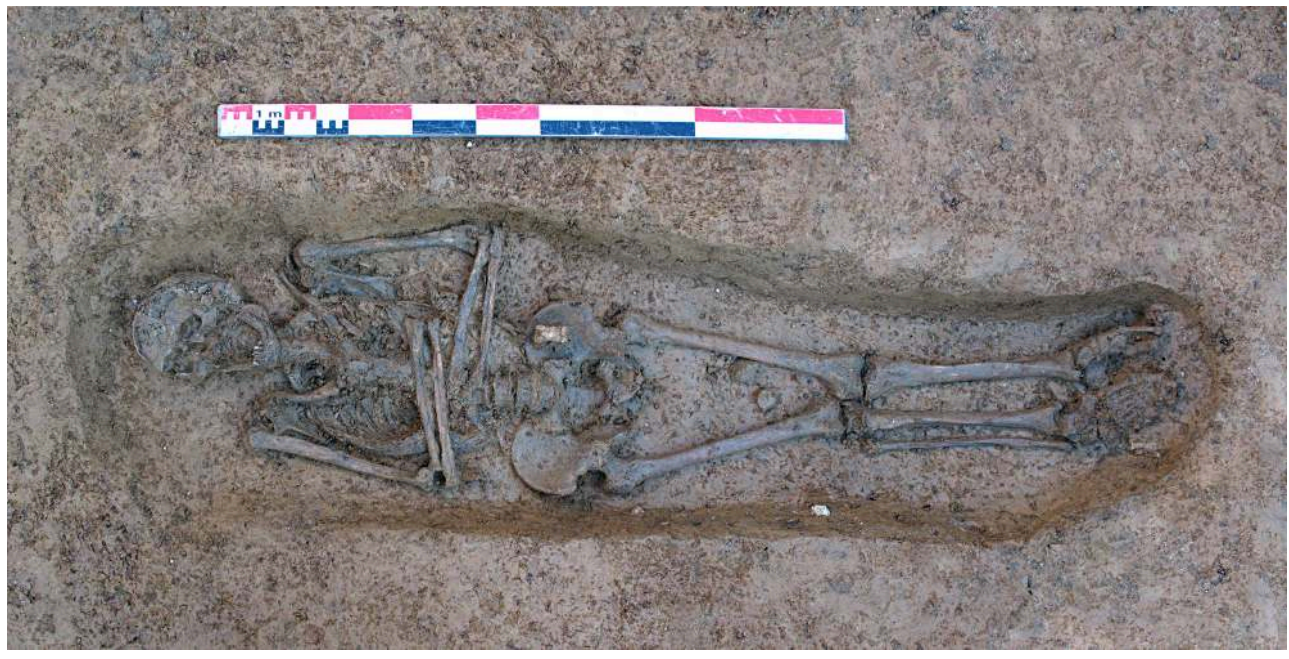

Fig. 4 - Gigny-sur-Suran, la sépulture présente dans le chœur de l'église (cl. D. Billoin, Inrap).

$5 \quad$ Ils sont composés de blocs bruts de calcaire mis à plats et liés à un mortier sableux de couleur beige, renfermant quelques charbons de bois épars qui emplissent un creusement d'une vingtaine de centimètres dans le substrat. L'un de ces massifs sert d'appui, en second lieu, à une élévation large de $0,90 \mathrm{~m}$, comportant deux étapes de construction : un premier parement régulier prend appui sur ce massif de maçonnerie, bien assisé, alors qu'un second, beaucoup plus irrégulier, lui est postérieur. Cette élévation correspond au mur gouttereau nord de la nef de l'église construite en 1780, observé sur une longueur de $3 \mathrm{~m}$. Un claveau en réemploi est intégré à la face interne de ce mur, constituant vraisemblablement la fondation d'un pilier supportant la voûte de la nef, et peut être mis en parallèle avec un autre départ de pilier mis au jour lors des sondages de 1986 . 
6 De par leur implantation, leurs dimensions et leur position stratigraphique, ces deux massifs de maçonnerie peuvent être interprétés comme des semelles de fondation des piles de l'église initiale. Leur espacement de l'ordre de $6 \mathrm{~m}$ est compatible avec les dimensions des édifices romans jurassiens des $\mathrm{XI}^{\mathrm{e}}$-XIII ${ }^{\mathrm{e}}$ siècles, à l'exemple de l'église de Saint-Lupicin. Cette observation renvoie à un plan classique de type basilical à trois vaisseaux, dont la largeur peut être estimée à environ $14 \mathrm{~m}$, suivant un rapport de $2 / 3$ entre la nef centrale et les bas-côtés. Des aménagements maçonnés plus récents sont également attestés, en relation soit avec la transformation de l'église en halles, soit des caniveaux réutilisant des fragments de pierres tombales des XVII ${ }^{\mathrm{e}}$ et XVIII ${ }^{\mathrm{e}}$ siècles.

Une sépulture est découverte sensiblement entre les deux piles de l'église primitive, dans le chœur de l'édifice, à $1,90 \mathrm{~m}$ de profondeur. Orientée est- ouest, la fosse est ovoïde, étroite et adaptée au corps. Son comblement est très argileux, homogène et compact, très proche de l'aspect du sédiment encaissant. L'individu est allongé sur le dos, les membres supérieurs repliés sur la poitrine, les membres inférieurs en extension, genoux et pieds resserrés (fig.5). L'examen taphonomique indique un colmatage différé du volume du corps, avec des constrictions suggérant l'utilisation d'un linceul, dans une fosse vraisemblablement couverte de planches en bois. L'analyse biologique reconnaît un homme âgé de 30 à 45 ans lors de son décès, mesurant entre 1,69 et 1,74 $\mathrm{m}$. De nombreuses lésions caractéristiques de la maladie hyperostosique diffuse idiopathique, appelée aussi maladie de Forestier, ont été relevées, alors que la tubérosité tibiale gauche est marquée par un véritable écrasement, à mettre en relation avec une position prolongée à genoux, peut-être de prière. L'étiologie de l'hyperostose diffuse est inconnue, mais des travaux ont retenu une hypothèse occupationnelle de type "monastic way of life", correspondant à une sédentarité et à un régime alimentaire riche ${ }^{6}$. La localisation de cette sépulture, isolée au sein de l'édifice, corrobore l'interprétation d'une tombe de prêtre. À noter une phalange de bœuf reposant au contact de l'ilion gauche du défunt, un dépôt intentionnel - symbolique, amulette ou autres pratiques -, dont la nature exacte ne pourra être comprise que par le biais de données comparatives.

Une datation radiocarbone des ossements de cette sépulture indique un enfouissement entre les années 901 et 1031 (Ly-14199). Elle offre, du même coup, une datation beaucoup plus précoce à cette église paroissiale, rejoignant la problématique des doubles édifices religieux sur Gigny, comme on en connaît ailleurs dans le Jura.

\section{NOTES}

1. C. SAPIN et S. BULlY, "Les abbayes Saint-Pierre de Gigny et Saint-Pierre de Baume (Jura)», Bucema, 13 (2009), p. 54-56.

2. Y. JEANIN, « Gigny, ancienne église paroissiale », Gallia, 1988, p. 102 ; C. SAPIN, « Gigny », in Éclats d'histoire. Dix ans d'archéologie en Franche-Comté, Besançon, 1995, p. 372-373.

3. A. ROUSSET, Dictionnaire géographique, historique et statistique des communes de Franche-Comté et des hameaux qui en dépendent, t. 3, Besançon, 1855, p. 246. 
4. A. ROUSSET, Dictionnaire géographique..., ibid.

5. D. BILloIN, Gigny-sur-Suran (39). Rue Abbé Bernon, rapport de diagnostic archéologique, Inrap, 2007, $24 \mathrm{p}$.

6. N. HUBERT, D. BILLOIN, S. GIGNARD, J.-L. CHOPARD et L. TATU, «À propos d'un cas d'hyperostose diffuse idiopathique du squelette chez un homme d'église de l'an Mil », à paraître.

INDEX

Mots-clés : église paroissiale

Index géographique : France/Gigny-sur-Suran 